\title{
REFERENCES
}

1. L. Auslander, On the Euler characteristic of compact locally affine spaces, Comment. Math. Helv. vol. 35 (1961) pp. 25-27.

2. L. Auslander and L. Markus, Holonomy of fat affinely connected manifolds, Ann. of Math. vol. 62 (1955) pp. 139-151.

3. H. Zassenhaus, Beweis eines Satzes ïber discrete Gruppen, Abh. Math. Sem. Univ. Hamburg vol. 12 (1938) pp. 289-312.

YALE UNIVERSITY AND

INDIANA UNIVERSITY

\section{A GENERAL CLEBSCH-GORDAN THEOREM}

BY ROBERT STEINBERG

Communicated by N. Jacobson, March 17, 1961

Relative to a Cartan decomposition of a simple Lie algebra over the complex field and an ordering of the roots, let $W$ be the Weyl group, $\phi$ half the sum of the positive roots, and $P(\beta)$ the number of partitions of $\beta$ as a sum of positive roots. In a fairly complicated way, Kostant [2] has proved that the multiplicity of $\mu$ as a weight in the irreducible representation with highest weight $\lambda$ is

$$
m_{\lambda}(\mu)=\sum_{s \in W} \operatorname{det} s P(s(\phi+\lambda)-(\phi+\mu)) .
$$

Cartier [1] and the present author have noticed, independently, that Weyl's character formula and (1) are simple formal consequences of each other. (Incidentally, Cartier seems to be wrong in saying that Kostant's work thus provides another algebraic proof of Weyl's formula, since the latter is Kostant's starting point for the proof of (1).) In this note we deduce from (1) the following explicit formula for the multiplicity of an irreducible representation in the tensor product of two others. If the algebra is of type $A_{1}$, the result is the classical Clebsch-Gordan Theorem.

THEOREM. Let $\pi_{\lambda}$ be the irreducible representation with highest weight $\lambda$. Then the multiplicity of $\pi_{\delta}$ in $\pi_{\beta} \otimes \pi_{\gamma}$ is 


$$
m(\beta, \gamma ; \delta)=\sum_{r, s \in W} \operatorname{det} r s P(r(\phi+\beta)+s(\phi+\gamma)-(2 \phi+\delta)) .
$$

To prove this, we use Weyl's formula to write

$$
\begin{aligned}
\sum_{\mu} m_{\beta}(\mu) \exp \mu \sum_{s \in W} \operatorname{det} s \exp [s(\phi+\gamma)] \\
\quad=\sum_{\eta} m(\beta, \gamma ; \eta) \sum_{s \in W} \operatorname{det} s \exp [s(\phi+\eta)] .
\end{aligned}
$$

Here $\mu$ runs over the set of weights and $\eta$ over the set of highest weights. A comparison of the coefficients of $\exp (\phi+\delta)$ yields

$$
m(\beta, \gamma ; \delta)=\sum_{s \in W} \operatorname{det} s m_{\beta}(\phi+\delta-s(\phi+\gamma)),
$$

and then by (1), the theorem.

\section{REFERENCES}

1. P. Cartier, On H. Weyl's character formula, Bull. Amer. Math. Soc. vol. 67 (1961) p. 228-230.

2. B. Kostant, $A$ formula for the multiplicity of a weight, Trans. Amer. Math. Soc. vol. 93 (1959) pp. 53-73.

University of California, Los Angeles 